\title{
Reflets
}

Revue ontaroise d'intervention sociale et communautaire

\section{La formation en promotion de la santé en Ontario français}

\section{Francine Deroche}

Volume 1, numéro 1, printemps 1995

Des pratiques à notre image : défis et ressources

URI : https://id.erudit.org/iderudit/026071ar

DOI : https://doi.org/10.7202/026071ar

Aller au sommaire du numéro

Éditeur(s)

Reflets : Revue ontaroise d'intervention sociale et communautaire

ISSN

1203-4576 (imprimé)

1712-8498 (numérique)

Découvrir la revue

Citer cet article

Deroche, F. (1995). La formation en promotion de la santé en Ontario français. Reflets, 1(1), 238-244. https://doi.org/10.7202/026071ar

Tous droits réservés (C) Reflets : Revue ontaroise d'intervention sociale et communautaire, 1995
Ce document est protégé par la loi sur le droit d'auteur. L'utilisation des services d'Érudit (y compris la reproduction) est assujettie à sa politique d'utilisation que vous pouvez consulter en ligne.

https://apropos.erudit.org/fr/usagers/politique-dutilisation/ 


\section{La formation en promotion de la santé en Ontario français}

Francine Deroche*

Coordonnatrice

Volet francophone du cours d'été en promotion de la santé de l'Ontario

\section{Introduction}

La promotion de la santé, qui s'inscrit dans la pratique d'un nombre croissant d'intervenantes et d'intervenants communautaires, se veut un processus visant à faciliter, pour les personnes qui s'y engagent, une plus grande maitrise des facteurs ayant une incidence sur leur santé. Parmi ces facteurs, on retrouve, entre autres, les conditions de vie et de travail, le soutien de la famille, des amies et des amis et l'environnement. (Conseil du premier ministre sur la santé, le bien-être et la justice sociale, 1993).

Plusieurs organismes ${ }^{1}$ ont uni leurs efforts en 1993 pour mettre sur pied le premier cours d'été en promotion de la santé en Ontario, subventionné par le Bureau de promotion de la santé et du développement social de Santé Canada et la Direction de la promotion de la santé du ministère ontarien de la Santé. Reconnaissant les besoins particuliers des Franco-Ontariennes et FrancoOntariens, un comité consultatif a été créé afin d'organiser une version française de ce cours. La Fondation de la recherche sur la toxicomanie, le Centre ontarien d'information en prévention, la Direction de la promotion de la santé du ministère ontarien de la Santé, le Bureau des services en français du ministère ontarien de la Santé, le Centre médico-social communautaire de Toronto, le Regroupement des intervenants et intervenantes francophones 
en santé et services sociaux de l'Ontario et le ministère ontarien de l'Éducation et de la Formation ont collaboré à la mise sur pied de la composante francophone du cours d'été 1994. Ce cours présente une façon novatrice de regrouper la théorie et la pratique en promotion de la santé.

\section{Besoins de formation}

Afin de mieux cerner les besoins de formation des intervenantes francophones, le comité consultatif francophone a décidé de mener une étude (Larocque, 1994) à l'échelle provinciale auprès de 486 intervenantes et intervenants qui avaient démontré un intérêt à la promotion de la santé.

Les 208 répondantes et répondants (retour de 43 pour cent) étaient majoritairement des femmes âgées de 26 à 45 ans qui détenaient un diplôme universitaire en éducation, en sciences infirmières, en service social, en psychologie ou en art (par ex., philosophie, sociologie, histoire). Notons qu'il y avait une bonne représentativité régionale au sein de l'échantillon.

Alors que 82 pour cent des répondantes et répondants anticipent offrir des services en promotion de la santé, 60 pour cent indiquent qu'ils n'ont pas la formation requise pour offrir ces services.

Les répondantes et répondants ont reconnu l'importance de plus de 50 différents sujets reliés à la promotion de la santé. Par contre, sept (7) de ces sujets ont retenu l'attention de plus de 10 pour cent d'entre eux. Parmi ces sujets, trois (3) portent sur les approches globales, c'est-à-dire la planification et l'évaluation de programmes, le développement communautaire et l'analyse de besoins. Les quatre (4) autres traitent de contenus précis, soit les compétences parentales, la nutrition, la toxicomanie et le tabagisme.

Quant aux approches pédagogiques, les répondantes et répondants ont mentionné qu'ils préferent les activités concrètes 
Reflets

plutôt qu'abstraites, et les activités de groupe plutôt qu'individuelles. Des approches telles que le développement d'outils de travail, les présentations d'expériences communautaires, les mises en pratique et les discussions de groupe sembleraient donc susceptibles de les intéresser. D'après ces résultats, Larocque (1994) recommande également l'utilisation de courtes présentations théoriques qui font le lien avec les sections plus expérimentales et des écrits pouvant servir de référence durant et après la formation.

Soixante-quatorze pour cent des répondantes et répondants sont en faveur de la formation à distance car celle-ci est accessible, équitable, pratique, facile et peu coûteuse. Par contre, on reconnaît l'importance de la planification et de la conception pédagogique afin d'assurer le bon fonctionnement de telles activités.

Quant à l'aspect financier, comme plusieurs francophones travaillent dans des services avec des ressources limitées, les activités de formation devraient être offertes au plus bas coût possible. Il s'avère aussi important que certains services soient subventionnés afin d'assurer la participation du plus grand nombre possible de personnes.

En plus de mettre en évidence le besoin réel de formation en promotion de la santé pour les intervenantes et intervenants franco-ontariens, l'étude offre des pistes concrètes pour répondre à ce besoin.

\section{Cours d'été 1994}

Le cours d'été en promotion de la santé établit les premiers jalons d'une démarche de formation pour les intervenantes et intervenants francophones. Le cours a pour objectif principal de les outiller afin d'être en mesure d'oeuvrer dans le domaine de la promotion de la santé dans leurs communautés respectives et ce, en français. Ses objectifs spécifiques sont de permettre aux personnes inscrites:

- de se familiariser avec les concepts de base et les stratégies de promotion de la santé; 
- d'acquérir des connaissances et des habiletés dans la planification et la mise en pratique de stratégies de promotion de la santé;

- de mettre en pratique les concepts et les stratégies de promotion de la santé au niveau de l'intervention dans les différents milieux; et

- de créer de nouveaux liens et d'élargir les réseaux existants de personnes oeuvrant en promotion de la santé.

Du 8 au 10 juin 1994, dix-huit (18) intervenantes et intervenants francophones provenant de toutes les régions de la province ont participé au premier cours, qui offrait des concepts de base ainsi que des exemples pratiques. Les participantes et participants ont aussi eu l'occasion de mettre en pratique de nouvelles connaissances et expériences acquises.

Deux professeurs ont abordé les concepts de promotion de la santé et de développement communautaire ainsi que les étapes dans la planification de programmes. Divers groupes communautaires, provenant de Cornwall, Sudbury, Vanier et PrescottRussell, ont présenté des exemples pratiques de programmation axée sur le développement communautaire ainsi que des exemples de programmes traitant de contenus précis tels que les compétences parentales et la toxicomanie.

Une session additionnelle avait pour but de discuter de l'importance du mouvement de Communautés en santé pour les communautés francophones de l'Ontario.

\section{Résultats de l'évaluation}

D'après les résultats de l'évaluation préliminaire effectuée quotidiennement pendant la durée du cours et du suivi téléphonique entamé en octobre 1994, les participantes et participants ont été très satisfaits de la structure ainsi que du contenu du cours d'été. Les approches pédagogiques telles que les études de cas et la distribution de publications ont été très bien accueillies. Le 
Reflets

cours a eu un impact sur leur travail communautaire en les conscientisant et en leur offrant des outils pratiques.

Dans cette même évaluation, les participantes et participants ont identifié le besoin d'aborder certains sujets et stratégies lors d'un prochain cours d'été pour les personnes qui ont déjà acquis de l'expérience dans le domaine de promotion de la santé. Ces sujets et stratégies comprennent la formation de coalitions, la recherche et le recrutement de fonds, le travail en milieu minoritaire et le marketing social. L'importance d'accorder beaucoup de temps à la pratique plutôt qu'à la théorie (quoique celle-ci s'avère nécessaire) a été soulignée. On insiste sur l'importance d'avoir d'autres activités de formation en promotion de la santé à l'avenir, y compris l'offre d'ateliers régionaux et la préparation de publications et de documents de lecture. Un cours d'été en 1995 serait utile dans la mesure où le contenu offert apporte des éléments nouveaux. On suggère également la pertinence d'offrir le cours à l'extérieur de Toronto dans les années à venir.

Somme toute, les résultats de l'évaluation appuient les conclusions de l'étude de besoins en confirmant, notamment, l'existence d'un besoin réel de formation en promotion de la santé pour les francophones de l'Ontario. Le cours d'été semble combler, au moins en partie, ce besoin.

\section{Nouveautés pour le cours d'été 1995}

Suite à l'expérience de 1994, le comité organisateur a pu tirer plusieurs conclusions ainsi qu'apporter des changements nécessaires. Entre autres, le comité organisateur pour le cours d'été en promotion de la santé 1995 compte maintenant une représentation régionale, assurée par trois personnes ayant participé au cours d'été 1994. Les membres du comité organisateur sont : le Centre ontarien d'information en prévention, la Direction de la promotion de la santé du ministère ontarien de la Santé, le Service de santé publique de Sudbury \& district, le ministère de l'Éducation 
et de la Formation de l'Ontario, le Centre médico-social communautaire de Toronto et le Service de la santé de la municipalité régionale d'Ottawa-Carleton. Afin de mieux répondre aux divers besoins d'apprentissage identifiés dans l'évaluation, les personnes participant au cours d'été 1995 auront le choix d'assister au cours I (principes de base) ou au cours II (stratégies telles que le marketing social et la formation de coalitions) ou encore, aux deux cours.

À long terme, le comité organisateur prévoit l'établissement de partenariats avec le réseau collégial franco-ontarien afin d'étudier la possibilité d'offrir une formation à distance ainsi que des crédits universitaires. La possibilité d'offrir le cours d'été dans différentes régions de la province est aussi à l'étude.

\section{Conclusion}

L'avantage à long terme du cours d'été en promotion de la santé est de former des intervenantes et intervenants franco-ontariens capables d'oeuvrer en français dans leur communauté. Ces personnes sauront assurer la relève au niveau de la formation, des connaissances et des compétences en promotion de la santé. Il s'avère donc critique de rester à l'affût des besoins exprimés en instaurant un processus d'évaluation continue. Ce processus permettra d'apporter les changements nécessaires afin de continuer à répondre aux besoins de formation réels en Ontario français.

\section{Notes}

* Veuillez faire parvenir toute correspondance a/s du Centre ontarien d'information en prévention, 415, rue Yonge, bureau 1 200, Toronto (Ontario) M5B 2E7.

No de téléphone: (416) 408-2121 poste 268.

No de télécopieur: (416) 408-2122 
Reflets

1. Les organismes engagés comprennent le Centre for Health Promotion de l'université de Toronto, l'Institut des études pédagogiques de l'Ontario, le Centre ontarien d'information en prévention, le Bureau de promotion de la santé et de développement social de Santé Canada, la Direction de la promotion de la santé et la Direction de la santé publique du ministère ontarien de la Santé, la Fondation de la recherche sur la toxicomanie, le East York Health Unit, le Waterloo Health Unit/l'Association de la santé publique,l'Université polytechnique de Ryerson, le Regional Women's Health Centre (Women's College Hospital) et l'Université de York.

\section{Bibliographie}

CONSEIL DU PREMIER MINISTRE SUR LA SANTÉ, LE BIEN-ÊTRE ET LA JUSTICE SOCIALE (1993). Prendre soin de la santé. Toronto: L'Imprimeur de la Reine de l'Ontario.

LAROCQUE, Daniel (1994). Rapport final: Les besoins de formation de la communauté franco-ontarienne en promotion de la santé. 